\title{
Green Synthesis of Silver - Nanocomposite for Treatment of Textile Dye
}

\author{
Priya Banerjee ${ }^{1}$, Shubhra Sau ${ }^{1}$, Papita Das ${ }^{2 *}$ and Aniruddha Mukhopadhyay ${ }^{1}$ \\ ${ }^{1}$ Department of Environment Science, University of Calcutta, Kolkata, India \\ ${ }^{2}$ Department of Chemical Engineering, Jadavpur University, Kolkata, India
}

Received: March 17, 2014; Accepted: June 02, 2014; Published: June 05, 2014

*Corresponding author: Papita Das, Department of Chemical Engineering, Jadavpur University, Kolkata, India, Email: papitasaha@gmail.com

\begin{abstract}
Textile industries not only consume huge volumes of water but also generate an equally alarming quantity of toxic dye rich waste water. This waste water if treated efficiently may be further reused for domestic and industrial purposes. Hence it is essential to develop, a cost effective and environmentally benign technique for adsorption of toxic dyes from textile effluents. Firstly, Ocimum tenuiflorum (Black Tulsi) leaf extract mediated green synthesis of silver nanoparticles (AgNPs) was carried out. The AgNPs were characterized using SEM and TEM analysis. These AgNPs were further utilized for preparing silver - nanocomposites with soil and applied as a novel nanocomposite for adsorption of reactive dye. However, this nanocomposite was determined to possess higher efficiency in comparison to soil as adsorbent for the removal of reactive turquoise blue dye under same experimental conditions. Moreover using these Ag-nanocomposites as adsorbent helped in achieving about $96.8 \%$ removal of reactive turquoise blue dye from effluent solution.
\end{abstract}

Keywords: Silver Nanoparticles; Nanocomposites; Reactive Dye; Textile Effluent; Treatment

\section{Introduction}

Rapid growth of various industries paralleling the swift rise in civilization and modernization is raising a recent global concern by generating huge volumes of toxic effluents. Disposal of these toxic effluents have lead to a severe contamination of the adjacent water bodies in turn causing relentless harm to life in and around them. Such effluents frequently rich in toxic dyes are often discharged into the environment without proper preceding treatments and result in an increased burden of health care issues in a developing country like India and worldwide. Of the many hazardous dyes used in textile industries, one of significance is reactive turquoise blue. Reactive dyes are highly soluble in nature and thereby, the total quantity of dye in solution is not taken up by the textile fibres [1]. Hence inefficient textile processing may result in $10-15 \%$ of such dyes being discharged directly along with wastewater into the surrounding environment [2].

These dyes often exhibit lethal effect and may prove to be genotoxic, mutagenic, and carcinogenic to plants and animals alike [3]. Being incessantly discharged along with industrial effluents, reactive dye removal is one of the most alarming issues faced while addressing environmental pollution [4-7]. Azo, nitro or sulfo moieties present in these dyes make them recalcitrant to microbial degradation as a result of which they accumulate in the biota [8]. Reactive dye exposure may result in health hazards like cancer, methaenoglobinaemia, extreme inflammatory responses in GI tract, irritation in the eyes, cytogeno-toxicity (acts as mitotic poison), etc.

Hence, it is becoming imperative to ensure the proper treatment of wastewater prior to discharge thereby making it a vital objective to prevent environmental contamination by innovating novel preventive and remedial measures with wide applications. Recent applications of nanotechnology, bio-nanotechnology, bioprocessing, etc. for dye removal have gained much interest but their broad implementation is yet to be achieved. A stride towards making these attempts successful is to execute them in an economic, convenient and sustainable way of dye removal from industrial wastewater. Successful application of the previously mentioned concepts depends upon improvisation of existing technologies. An example of the most universally utilized adsorbent is soil as it possesses unique physicochemical properties, structure, is highly available and inexpensive. Hence, it can be improvised and used as an effective adsorbent. In this study, concept of Nanotechnology has been implemented for designing a novel soil composite for removal of dye (Reactive Turquoise Blue) removal from industrial effluent.

In the present study, soil has been combined with silver nanoparticles (AgNPs) prepared by bioreduction process. The bioreduction process was selected because of its cost effectiveness, less time required and accessibility of ingredients of the process [9-14]. Here, Ocimum tenuiflorum (Black Tulsi) leaf extract is used as reducing and capping agent owing to wide availability across the country throughout the year and its cost efficiency. Leaves of this plant are widely consumed in India for its reported antibacterial and medicinal properties. It also has reported anticancer, antidiabetic, analgesic, adaptogenic and diaphoretic properties [15-17]. The chief ingredients of the leaf extract are triterpenes, flavonoids and eugenol, which are vital 
reducing and capping agents for nanoparticle (NP) formation [15, 18-21]. Hence, Ocimum tenuiflorum leaf extract was selected for bioreduction to AgNPs. Of all metal NPs, AgNPs were selected due to their simple, cost effective and green route of preparation. AgNPs possess antimicrobial properties [22] and are found to remain stable even after a month from its preparation [23].

In the next step, soil (clay) was charged with these bioreduced AgNPs to develop a novel green synthesized Ag-nano composite to be implemented as a successful adsorbent for efficient removal of toxic reactive dyes present in industrial effluent. Results revealed this nano-composite to possess excellent potential of toxic dye adsorption in laboratory scale. All experimental parameters were pre optimized with the help of batch experiments.

\section{Materials and Methods}

\section{Collection of Leaf Samples and Preparation of Extract}

Fresh Tulsi leaves (Ocimum tenuiflorum) of good health were collected from a nearby farm. They were rigorously washed to get rid of dust particles and then rinsed thrice with double distilled water and weighed afterwards. $10 \mathrm{~g}$ of collected leaves were finely chopped and crushed in $100 \mathrm{~mL}$ double distilled water and filtered for removal of pigments and unwanted particulate matter. This filtrate was further used for AgNP synthesis.

\section{Synthesis of silver nanoparticles}

$5 \mathrm{~mL}$ of aqueous leaf extract was added to $95 \mathrm{~mL}$ of $1 \mathrm{mM}$ silver nitrate solution taken in an Erlenmeyer flask. Due to sensitivity of silver nitrate towards light, the reaction was carried out in darkness. The color change of the reaction mixture was carefully monitored at every step of the procedure. Bioreduction of the mixture was completed in a microwave oven at a power level of $300 \mathrm{~W}$ in duration of $5 \mathrm{~min}$. Throughout the reaction procedure, the absorption maxima of the mixture was recorded after every $30 \mathrm{~min}$, using a spectrophotometer, till the color of the solution changed from pale light green to colloidal brown. The resultant colloidal solution was cooled to ambient temperature and preserved overnight to ensure complete bioreduction and saturation. The solution was then stored carefully for further use. Control samples were also analyzed at every step of the whole procedure.

\section{Characterization of silver nanoparticles}

Formation of AgNPs was confirmed by monitoring the periodic samples collected from the colloidal mixture solution by UV-Visible spectrophotometer at around $425 \mathrm{~nm}$. The AgNPs were characterized using SEM and TEM analysis.

\section{Preparation of silver nanocomposite}

For nano-composite preparation, a pre-weighed amount of soil was taken, churned to fine particles, sieved to remove impurities and added slowly to the nanoparticle solution prepared previously and mixed properly for nanocomposite adsorbent preparation. The composite mixture solution was set aside overnight in a shaker incubator for the soil particles to get appropriately coated by AgNPs. After 24h incubation with a constant speed of agitation the solution was kept for settling of the nano-composite. Following the settling of the prepared composite, supernatant was decanted and discarded and the composite mixture obtained was dried, finely churned and sieved again to obtain Ag-nanocomposites to be used for efficient dye removal.

\section{Adsorbate solution}

Reactive turquoise blue dye was obtained from a nearby textile unit $\left(\lambda_{\max }: 629 \mathrm{~nm}\right.$ ). Precise quantities of solid dye were dissolved in double distilled water to prepare the stock solutions of reactive dye. Solutions of various concentrations of dye were obtained by diluting this stock solution. The $\mathrm{pH}$ of the prepared solutions was adjusted by using $\mathrm{HCl}$ and $\mathrm{NaOH}$ solutions of 0.1 (N) strength respectively.

\section{Nano-composite characterization}

SEM and FTIR analysis was carried out to characterize the surface morphology and functional groups present in the AgNPSoil Nanocomposites and respectively both prior and post adsorption.

For SEM analysis, the samples were gold coated (JEOL, Japan and Model No. JFC-1600) prior to analysis and the images were obtained using Scanning Electron Microscope (ZEISS EVO-MA 10, Germany). The image details (like applied voltage, magnification, and size of the samples, etc) were incorporated in the images itself.

$\mathrm{KBr}$ pellet method of FTIR analysis of the dried Ag NPs was carried out using Jasco FT/IR-6300 Fourier Transform Infrared Spectrometer with a resolution of $4 \mathrm{~cm}^{-1}$ in transmittance mode (JASCO, Japan).

Scanning electron microscopy revealed the surface morphology of the nano-composite. The study was conducted using a scanning electron microscope (Model Hitachi S-3000N) at an electron acceleration voltage of $20 \mathrm{kV}$. The samples were coated with a thin layer of gold using a sputter coater to make them conductive and then used for scanning.

\section{Batch experiments for optimization}

$100 \mathrm{~mL}$ of dye solution of different concentrations were used for batch experiments. Parameters optimized included concentration of dye solution (20 - $100 \mathrm{ppm})$, weight of adsorbate $(0.25-1 \mathrm{~g} / \mathrm{L}), \mathrm{pH}$ (2.0 to 10.0$)$ and temperature (293, $298,303$ and $308 \mathrm{~K})$. The samples were incubated with a constant speed of agitation (180 rpm) (Model Innova 42, New Brunswick Scientific, Canada). Samples were collected for analyzing the residual dye present in the solution. The residual amount of dye was investigated using UV/VIS spectrophotometer (Shimadzu UV -1601). The quantity of dye adsorbed/unit nano-composite was estimated according to a mass balance equation (1):

$$
q_{e}=\frac{\left(C_{i}-C_{e}\right) V}{m}
$$

Where $C_{i}=$ initial dye conc. (mg/L), $C_{e}=$ equilibrium dye conc. 
in solution (mg/L), $V=$ volume of the solution (L), and $m=$ mass of the nano-composite $(\mathrm{g})$. The removal percentage $(\%)$ of dye was determined by means of the following equation:

$$
\operatorname{Removal}(\%)=\frac{C_{i}-C_{e}}{C_{i}} \times 100
$$

The adsorption experiments were replicated thrice and results obtained are expressed as Mean \pm SD. The error obtained was found to be within $\pm 3.0 \%$. All the statistical calculations were carried out with Origin software (version 7.0383, USA).

\section{Results and Discussion}

\section{Characterization of AgNP and Soil Sample}

The AgNPs obtained from the reaction of aqueous leaf extract of Ocimum tenuiflorum and silver nitrate $(1 \mathrm{mM})$ established by the reaction mixture turning reddish brown in color after incubation while the controls experienced no color change at all. This reddish brown color may have developed as a result of excitation of surface plasmon vibrations. The formation of AgNPs was monitored by collecting samples at intervals of $15 \mathrm{mins}$ till the desired change in color of the solution was achieved and by analyzing the samples collected using UV-VIS spectroscopy. The highest absorbance peak obtained at around $425 \mathrm{~nm}$ after different time intervals confirmed the synthesis of AgNPs. SEM and TEM analysis of the AgNPs obtained are shown in Figure 1A and $B$ respectively. It was determined that most of the AgNPs obtained ranged between $20 \mathrm{~nm}$ to $40 \mathrm{~nm}$ in diameter while the average size was $32.58 \mathrm{~nm}$.

FTIR analysis (Figure not shown) showed was carried out in the range of $500 \mathrm{~cm}^{-1}-4000 \mathrm{~cm}^{-1}$. Prominent spectral bands were observed at $654 \mathrm{~cm}^{-1}, 772 \mathrm{~cm}^{-1}, 2364.2 \mathrm{~cm}^{-1}, 1034.5 \mathrm{~cm}^{-1}$ and $3384.05 \mathrm{~cm}^{-1}$ which may be attributed to the presence of $-\mathrm{C}-\mathrm{Cl}-$, $-\mathrm{C}-\mathrm{H}-,-\mathrm{C}-\mathrm{O}-$ and $-\mathrm{O}-\mathrm{H}-$ moieties in the sample respectively.

The soil samples was analyzed to exhibit pH 7.34, moisture content $24.6 \%$, cation exchange capacity 29.62 (mequiv/100 g dry soil) bearing a surface area of $58.7 \mathrm{~m}^{2} / \mathrm{g}$, with a percentage of clay, silt and sand in the ratio 6:16:1.

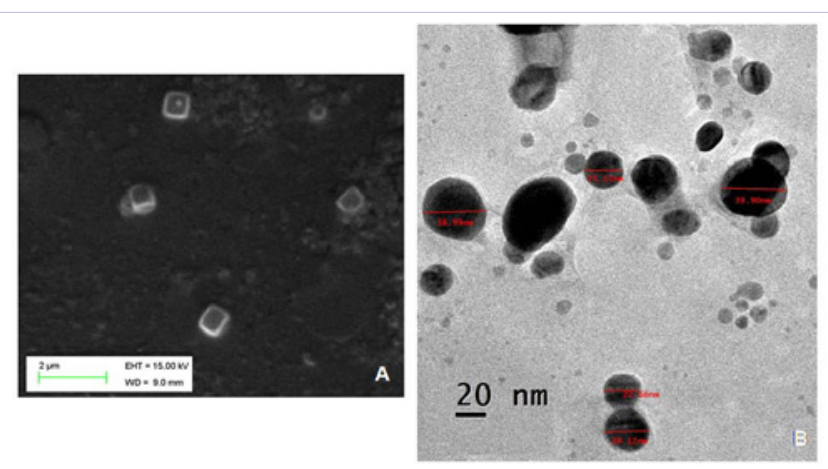

Figure 1: SEM (A) and TEM (B) analysis of AgNPs obtained from Ocimum tenuiflorum leaf extract.

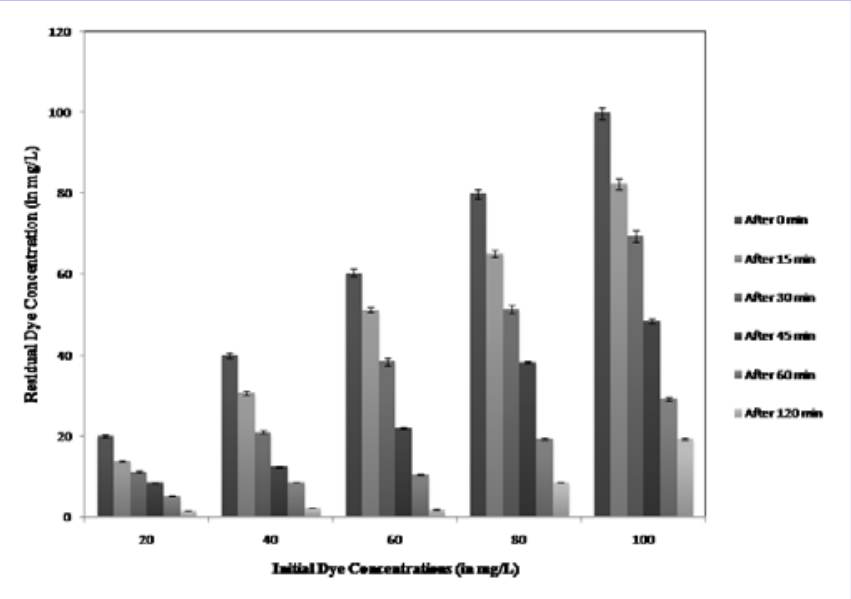

Figure 2: Effect of initial dye concentration on adsorption efficiency in a time-dependent manner.

\section{Estimation of Maximum Initial Dye Concentration Applicable}

Efficiency of the adsorbate developed is dependent upon the concentration of reactive blue dye present in solution initially. Experiments were carried out with $100 \mathrm{~mL}$ of each solution having reactive dye concentration 20, 40, 60, 80 and $100 \mathrm{mg} \mathrm{L}^{-1}$ and $\mathrm{pH}$ 6.5. Temperature was maintained at $298 \pm$ $2 \mathrm{~K}$ (room temperature). $0.5 \mathrm{~g} \mathrm{~L}^{-1}$ adsorbate was added to each of the solutions and the setup was maintained under constant speed of agitation. The amount of dye adsorbed per unit mass of the nanocomposite increased with increased in the initial dye concentration. As observed in Figure 2, the removal of dye increased and residual dye concentration decreased with initial dye concentration from 20 to $100 \mathrm{mg} \mathrm{L}^{-1}$.

This nature may be attributed to a corresponding increase in the driving force developing as a result of concentration gradient. Additionally, higher concentrations of reactive turquoise blue ions in solution may have surrounded the active sites of the resulting in elevated adsorption efficiency. This is also supported by other studies which showed an increase in adsorption capacity with increased initial dye concentrations [24]. However, much higher concentration ( $80 \mathrm{mg} \mathrm{L}^{-1}$ and above) of dye may have caused saturation of the active sites of the adsorbent leading to its decreased efficiency. Since adsorption efficiency reduced after increasing the concentration of initial dye to $80 \mathrm{mg} \mathrm{L}^{-1}$, hence all experiments were carried out with an initial dye concentration of $60 \mathrm{mg} \mathrm{L}^{-1}$.

\section{Estimation of Effective Ag-nanocomposite Dose}

Adsorption efficiency is known to have been influenced by adsorption hence making the adsorbent dose a vital factor for a particular initial concentration of the reactive dye solution under the experimental conditions. Dye solution containing $60 \mathrm{mg} \mathrm{L}^{-1}$ dye was taken and the $\mathrm{pH}$ of the solution was measured and controlled throughout the experiment at 6 . The temperature was fixed at $298 \pm 2 \mathrm{~K}$. The $\mathrm{pH}$ of the solution was measured for all the 
experiments before and after adsorption experiments and was found to remain unaltered. The experiment was performed until equilibrium was attained between the solution and the nanocomposite materials. Effect of nanocomposite (adsorbate) dose with time on dye removal efficiency is shown Figure 3. Results show an increase in adsorption efficiency from $80.6 \%$ to 96.8 $\%$ with an increase in the adsorbent dose from 0.25 to $1 \mathrm{~g} \mathrm{~L}^{-1}$ and hence all further experiments were carried out with $0.25 \mathrm{~g} \mathrm{~L}^{-1}$ of adsorbate. An increase in the available nano-composite surface area may be held responsible for an increase in the percentage of dye removal as already reported previously $[4,25]$.

\section{Estimation of Effect of Temperature}

Temperature is considered an important experimental parameter influencing adsorption potential and therefore batch experiments were carried out at different temperatures. Four solutions containing $60 \mathrm{mgL}^{-1}$ of reactive dye each was taken and the $\mathrm{pH}$ of the solution was adjusted to $6.0 .5 \mathrm{~g} \mathrm{~L}^{-1}$ adsorbate was added to each solution and incubated at 293, 298, 303 and $308 \mathrm{~K}$ respectively with constant agitation. Data obtained are presented in Figure4.

Figure 4 shows that all other conditions remaining constant, an increase in temperature led to an increase in the percentage of dye adsorption. This might be explained by a probable increase in affinity of binding sites of the adsorbent for dye molecules with a corresponding increase in temperature. Rise in temperature may also have resulted in a higher mobility of the dye molecules accompanied by a reduction in the retarding forces acting on the same thereby incrementing the adsorbent efficiency. This behavior suggested that the mechanism of dye adsorption by nanocomposite was endothermic in nature. A similar trend was reported for adsorption of CV onto treated ginger waste [26].

\section{Estimation of Effect of $\mathbf{p H}$}

$60 \mathrm{mgL}^{-1}$ of reactive dye containing solutions were taken and their $\mathrm{pH}$ was adjusted to 2, 4, 6, 8 and $10.0 .5 \mathrm{~g} \mathrm{~L}^{-1}$ adsorbate

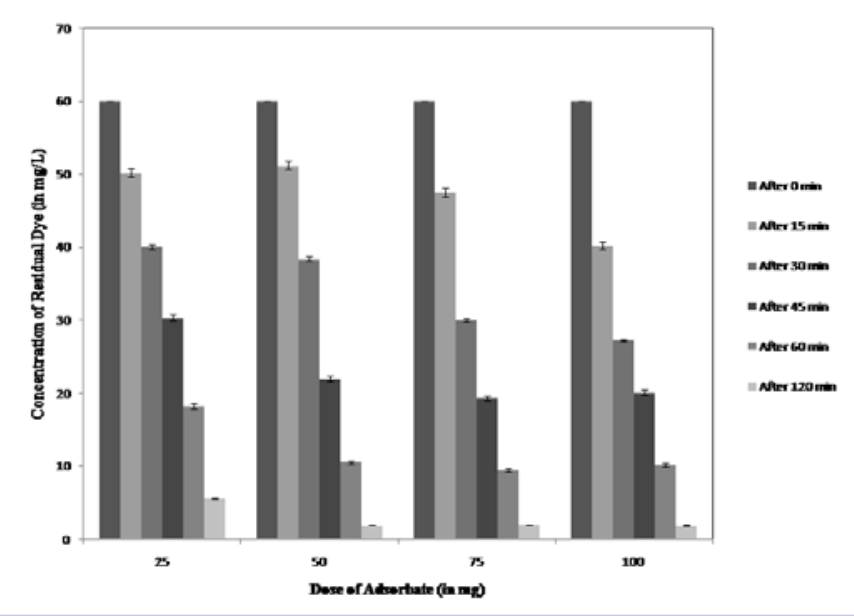

Figure 3: Effect of adsorbate dose on adsorption efficiency in a timedependent manner.

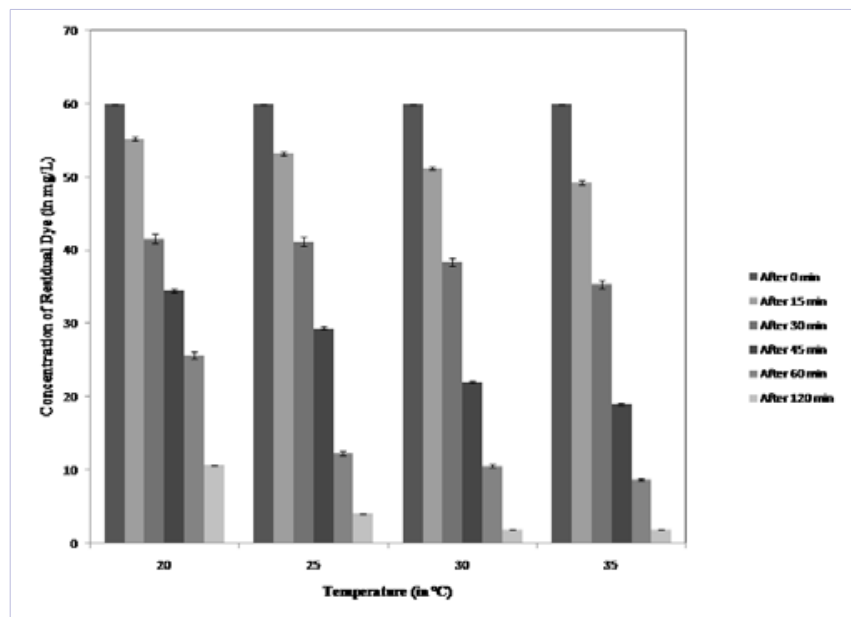

Figure 4: Effect of temperature on adsorption efficiency in a time-dependent manner.

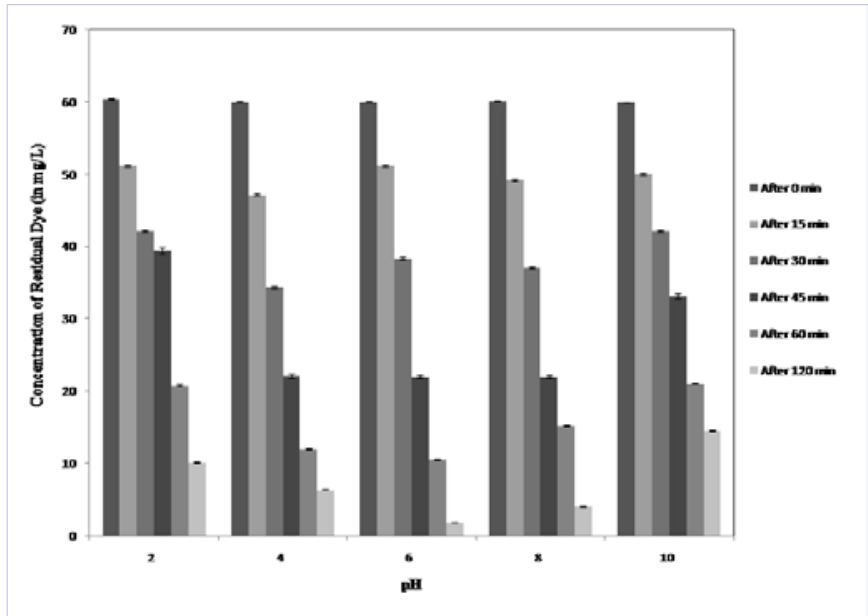

Figure 5: Effect of $\mathrm{pH}$ on adsorption efficiency in a time-dependent manner.

was added to each solution and all were incubated at $303 \pm 2 \mathrm{~K}$ respectively with constant agitation. Results obtained are shown in Figure 5.

Optimum adsorption efficiency was observed in solutions whose $\mathrm{pH}$ was adjusted at 6 . Hence, the same was maintained for all other experiments.

\section{Adsorbate Morphology Before And After Treatment}

SEM and EDS (inset) analysis of the nanocomposites used for dye removal under optimized conditions are shown in Figure 6A and $6 \mathrm{~B}$ respectively.

\section{Conclusion}

Ag-Nanocomposite has been successfully prepared and implemented for removal of reactive turquoise blue dye from its aqueous solution. Results show that utilization of $0.5 \mathrm{~g} \mathrm{~L}^{-1}$ of this adsorbate for minimum $2 \mathrm{~h}$ at room temperature is sufficient to remove $96.8 \%$ of dye content from a solution containing 60 $\mathrm{mg} \mathrm{L}^{-1}$ of the same. In totality, the results suggest achievement 

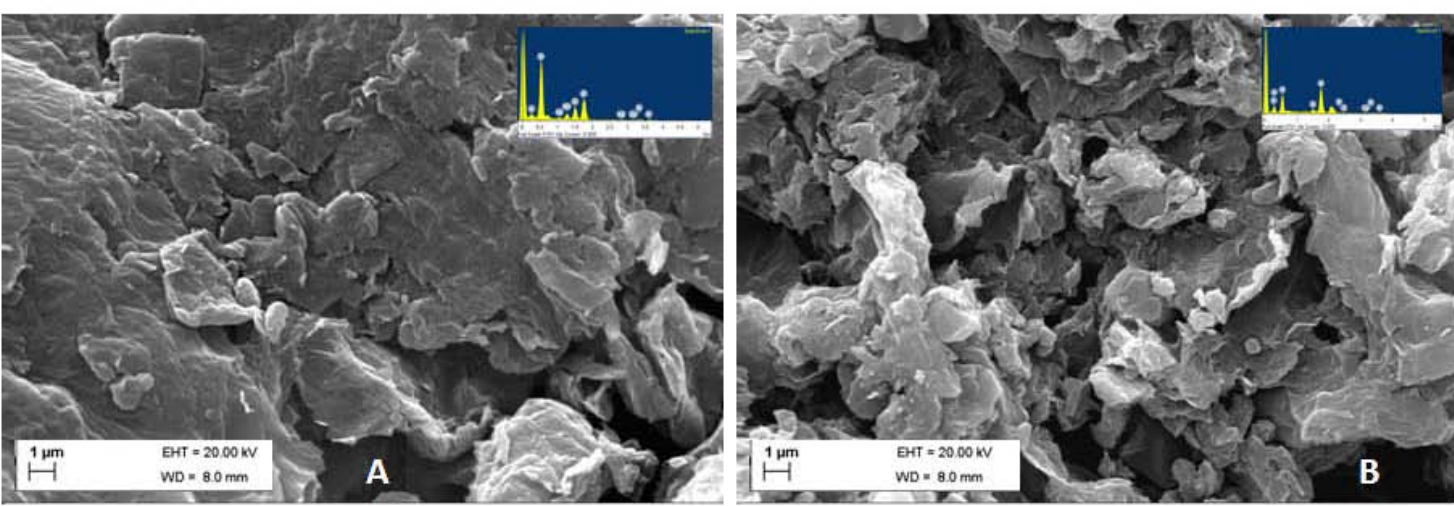

Figure 6: 6A and 6B showing surface morphology of Ag-Nanocomposite before and after reactive turquoise blue dye adsorption along with their EDS (inset) respectively.

of an environmentally sustainable, economically feasible and highly efficient adsorbent which may be considered useful for the removal of reactive dyes from effluent solution.

\section{Acknowledgements}

The authors are thankful to all members of Department of Environmental Science, University of Calcutta, Kolkata and Department of Chemical Engineering, Jadavpur University, Kolkata. We also acknowledge Mr. Tridip Sen and Miss Urmila Goswami, Center for Research in Nanoscience and Nanotechnology, University of Calcutta, for helping with SEM TEM and EDS analysis.

\section{References}

1. Ozsoy HD, Unyayar A, Mazmanci MA (2005) Decolourisation of reactive textile dyes Drimarene Blue X3LR and Remazol Brilliant Blue R by Funalia trogii ATCC 200800. Biodegradation 16(3): 195-204.

2. Kirby N, Marchant R, McMullan G (2000) Decolorisation of synthetic textile dyes by Phlebia tremellosa. FEMS Microbiol Lett 188(1): 93-96.

3. Puvaneswari N, Muthukrishnan J, Gunasekaran P (2006) Toxicity assessment and microbial degradation of azo dyes. Indian J Exp Biol 44 (8): 618- 626 .

4. Ahmad R (2009) Studies on adsorption of crystal violet dye from aqueous solution onto coniferous pinus bark powder (CPBP). J Hazard Mater 171(1-3): 767-773.

5. Akar ST, Ozcan AS, Akar T, Ozcan A, Kaynak Z (2009) Biosorption of a reactive textile dye from aqueous solutions utilizing an agro-waste. Desalination 249(2): 757-761.

6. Atmani F, Bensmaili A, Mezenner NY (2009) Synthetic textile effluent removal by skin almond waste. J Env Sci Technol 2: 153-169.

7. Saha S, Chattopadhyay D, Acharya K (2011) Preparation of silver nanoparticles by bio-reduction using Nigrospora oryzae culture filtrate and its antimicrobial activity. Dig J Nanomater Bios 6(4): 1519-1528.

8. Pourbabaee AA, Malekzadeh F, Sarbolouki MN, Najafi F (2006) Aerobic decolorization and detoxification of a disperse dye in textile effluent by a new isolate of Bacillus sp. Biotechnol Bioeng 93(4): 631-635.

9. El-Nour KMM, Eftaiha A, Al-Warthan A, Ammar RAA (2010) Synthesis and applications of silver nanoparticles. Arabian J of Chem 3(3): 135-140.

10. Guzman MG, Dille J, Godet S (2009) Synthesis of silver nanoparticles by chemical reduction method and their antibacterial activity. Int J Chem Biol Engg 2(3): 104-111.

11. Mouxing F, Qingbiao L, Daohua S, Yinghua L, Ning H et al. (2006) Rapid Preparation Process of Silver Nanoparticles by Bioreduction and Their Characterizations. Chinese J Chem Eng 14(1): 114-1 17.

12. Prabhu S and Poulose EK (2012). Silver nanoparticles: mechanism of antimicrobial action, synthesis, medical applications, and toxicity effects. Int Nano Lett 2: 32-42.

13. Sulaiman GM, Mohammad WH, Marzay TR, Al-Amiery AAA, Kadhum AAH et al. (2013) Green synthesis, antimicrobial and cytotoxic effects of silver nanoparticles using Eucalyptus chapmaniana leaves extract. Asian Pac J Trop Biomed 3(1): 58-63.

14. Xia Y, Xiong YJ, Lim B, Skrabalak SE (2009) Shape-Controlled Synthesis of Metal Nanocrystals: Simple Chemistry Meets Complex Physics? Angew Chem Int Ed 48 (1): 60-103.

15. Prakash P, Gupta N (2005) Therapeutic uses of Ocimum sanctum Linn (Tulsi) with a note on Eugenol and its pharmacological actions: A short review. Indian J Physiol Pharmacol 49(2): 125-131.

16. Seth J, Sood S, Seth S, Talwar A (2004) Evaluation of hypoglycemic and antioxidant effect of Ocimum sanctum. Indian J Clin Biochem 19(2): 152155 .

17. Rai V, Iyer U, Mani UV (1997) Effect of Tulasi (Ocimum sanctum) leaf powder supplementation on blood sugar levels, serum lipids and tissues lipids in diabetic rats. Plant Food Hum Nutr 50(1): 9.

18. Chathopadhyay RR, Sarkar SK, Ganguly S, Medda C, Basu TK (1992) Hepatoprotective activity of Ocimum sanctum leaf extract against paracetamol induced hepatic damage in rats. Indian J Pharmacol 24:63.

19. Uma Devi P, Ganasoundari A, Rao BSS, Srinivasan KK (1999) Invivo radioprotection by Ocimum flavonoids: Survival of mice. Radiat Res 151(1):74-78.

20. Philip D, Unni C (2011) Extracellular biosynthesis of gold and silver nanoparticles using Krishna Tulsi (Ocimum sanctum) leaf. Physica E 43(7): 1318-1322.

21. Mallikarjuna K, Narasimha G, Dillip GR, Praveen B, Shreedhar B et 
al. (2011) Green synthesis of silver nanoparticles using Ocimum leaf extract and their characterization. Digest Journal of Nanomaterials and Biostructures 6(1):181-186.

22. Choi O, Deng KK, Kim NJ, Ross L, Surampalli RY et al. (2008) The inhibitory effects of silver nanoparticles, silver ions, and silver chloride colloids on microbial growth. Water Res 42(12): 3066-3074.

23. Das R, Gang S, Nath SS (2011) Preparation and Antibacterial Activity of Silver Nanoparticles. J Biomater and Nanobiotechnol 2: 472-475.

24. Chowdhury S, Chakraborty S, Saha PD (2013) Response surface optimization of a dynamic dye adsorption process: A case study of crystal violet adsorption onto $\mathrm{NaOH}$-modified rice husk. Env Sci Polln Res Int 20(3):1698-1705.

25. Wang XS, Liu X, Wen L, Zhou Y, Li Z (2008) Comparison of basic dye crystal violet from aqueous solution by low-cost biosorbents. Sep Sci Technol 43(14): 3712-3731.

26. Kumar R, Ahmad R (2011) Biosorption of hazardous crystal violet dye from aqueous solution onto treated ginger waste (TGW). Desalination 265(1-3): 112-118. 